\title{
The Rayleigh Quotient Iteration and Some Generalizations for Nonnormal Matrices*
}

\author{
By B. N. Parlett
}

\begin{abstract}
The Rayleigh Quotient Iteration (RQI) was developed for real symmetric matrices. Its rapid local convergence is due to the stationarity of the Rayleigh Quotient at an eigenvector. Its excellent global properties are due to the monotonic decrease in the norms of the residuals. These facts are established for normal matrices. Both properties fail for nonnormal matrices and no generalization of the iteration has recaptured both of them. We examine methods which employ either one or the other of them.
\end{abstract}

1. History. In the 1870's John William Strutt (third Baron Rayleigh) began his mammoth work on the theory of sound. Basic to many of his studies were the small oscillations of a vibrating system about a position of equilibrium.

In terms of suitable generalized coordinates, any set of small displacements (the state of the system) will be represented by a vector $q$ with time derivative $\dot{q}$. The potential energy $V$ and the kinetic energy $T$ at each instant $t$ are represented by the two quadratic forms

$$
V=\frac{1}{2}(M q, q)=\frac{1}{2} q^{*} M q, \quad T=\frac{1}{2}(H \dot{q}, \dot{q})=\frac{1}{2} \dot{q}^{*} H \dot{q},
$$

where $M$ and $H$ are suitable symmetric positive definite matrices, constant in time, and $q^{*}$ denotes the transpose of $q$.

Of principal interest are the smallest natural frequencies $\omega$ and their corresponding natural modes which can be expressed in the generalized coordinates in the form $\exp (i \omega t) x$ where $x$ is a constant vector (the mode shape) which satisfies

$$
\left(M-\omega^{2} H\right) x=0 .
$$

Among other things, Lord Rayleigh showed that the frequency can be written in terms of the mode shape

$$
\omega^{2}=V / T=x^{*} M x / x^{*} H x,
$$

the ratio of the two energies.

Received August 10, 1973.

AMS (MOS) subject classifications (1970). Primary 65F15; Secondary 15A18.

Key words and phrases. Eigenvector, eigenvalue, iterative methods, Rayleigh Quotient, global convergence, nonnormal matrix.

* This work was supported by the Office of Naval Research under Contract No. N00014-69A-0200-1017. Reproduction in whole or in part is permitted for any purpose of the United States Government. 
Now, this ratio is well defined even when $x$ is not a mode shape. Let $v$ be any nonzero real vector. The Rayleigh Quotient of $v$ (with respect to $M$ and $H$ ) is defined by

$$
\rho(v)=v^{*} M v / v^{*} H v .
$$

Lord Rayleigh pointed out that, for any $v, \omega_{\min }^{2} \leqq \rho(v) \leqq \omega_{\max }^{2}$ and suggested a technique for improving an approximation to a mode shape.

With the advent of digital computers in the 1950's, Lord Rayleigh's technique for improving a good approximation was turned into a method for the automatic computation of eigenvectors from an arbitrary initial guess. The question of the convergence of this iterative process was discussed by Temple [7] in England and Crandall [2] in the U.S.A. In 1958/59, Ostrowski [4] published six articles giving a rigorous and detailed analysis of the local asymptotic behavior of the original method and some variants of it. He discussed both the symmetric and the nonsymmetric cases. In 1968 came Kahan's proof [5] of convergence, for almost all starting vectors, in the symmetric case. We continue the investigation.

In the body of this paper, we will not treat the general case (see [3] for that) but take $H=I$. Our interest is in understanding the behavior of the RQI when $M$ is not symmetric. However, we shall begin by consolidating what is known, and that mainly concerns symmetric and normal $M$.

2. Notation. Matrices will be denoted by capital latin letters, column vectors by small latin letters except for $i, j, k, l, m, n$ which denote indices. Small Greek letters are reserved for other scalars. The conjugate transpose of a vector $u$ is denoted by $u^{*}$ whether or not $u$ be real. Throughout, we will use the Euclidean norm, $\|u\|=\sqrt{u^{*} u}$.

Let $C$ be an arbitrary $n \times n$ complex matrix with spectrum $\left\{\lambda_{1}, \cdots, \lambda_{r}\right\}$ and normalized eigenvectors given by

$$
C x_{j}=\lambda_{j} x_{j}, \quad y_{j}^{*} C=\lambda_{j} y_{j}^{*}, \quad x_{j}^{*} x_{j}=y_{j}^{*} y_{j}=1, \quad j=1, \cdots, r .
$$

If $\lambda_{j}$ is multiple, then $x_{j}$ and $y_{j}$ will not be unique. If $C$ is defective, then the $\left\{x_{j}\right\}$ do not span the whole space. When $C^{*}=C$, then $y_{j}=x_{j}$.

3. Basic Properties of the Rayleigh Quotient.

Definition. The Rayleigh Quotient $\rho$ is the function which assigns to any nonzero complex vector $u$ the scalar quantity

$$
\rho(u)=u^{*} C \boldsymbol{d} / u^{*} u=\sum \sum c_{j k} \bar{u}_{j} u_{k} / \sum\left|u_{i}\right|^{2} .
$$

When it is necessary to emphasize the role of $C$, we write $\rho(u) \equiv \rho(u, C)$.

We begin by listing, without proof, some basic facts.

Homogeneity. $\rho(\alpha u, \beta C)=\beta \rho(u, C) ; \alpha, \beta \neq 0$.

Translation Invariance. $\rho(u, C-\alpha I)=\rho(u, C)-\alpha$.

Boundedness. As $u$ ranges over all nonzero column vectors, $\rho(u)$ fills out a region in the complex plane which is called the field of values (also numerical range) of $C$. See Marcus and Minc [10] for more information. 
For our purposes we need only the fact that this region is closed, bounded, and convex.

If $C=C^{*}$, then the field of values is the real interval bounded by the extreme eigenvalues $\left[\lambda_{\min }, \lambda_{\max }\right]$.

Stationarity. Over complex vectors $u$, the function $\rho(u)$ is not differentiable in the components $u_{j}, j=1, \cdots, n$. However, $\rho$ is rational in the $u_{j}$ and the $\bar{u}_{j}$ and its derivative at $u$ in the direction of the unit vector $v$ is defined by

$$
\rho^{1}(u ; v) \equiv \lim [\rho(u+t v)-\rho(u)] / t
$$

as $t \rightarrow 0$ through real values.

A standard calculation yields

$$
\rho^{1}(u ; v) \equiv v^{*}(C-\rho(u)) u+u^{*}(C-\rho(u)) v .
$$

Note that

$$
\rho^{1}(u ; i v)=-i v^{*}(C-\rho(u)) u+i u^{*}(C-\rho(u)) v .
$$

Consequently, $\rho^{1}(u ; v)$ vanishes for all directions $v$; that is, $\rho$ is stationary at $u$, if and only if

$$
(C-\rho) u=0, \quad u^{*}(C-\rho)=0^{*} .
$$

In other words, $u$ must be an eigenvector of $C$ and $C^{*} ; u=x_{j}=y_{j}$ in the notation of Section 2. So for normal $C\left(C C^{*}=C^{*} C\right)$, the eigenvectors of $C$ yield the stationary points of $\rho$.

Example. Let $J=\left(\begin{array}{ll}0 & 1 \\ 0 & 0\end{array}\right)$. The function $\rho(u, J)=\bar{u}_{1} u_{2}\left(\left|u_{1}\right|^{2}+\left|u_{2}\right|^{2}\right)^{-1}$ has no complex stationary points although $0 \leqq|\rho(u, J)| \leqq \frac{1}{2}$. Over real vectors, $\rho(u, J)$ has a maximum at $(1,1)^{*}$ and a minimum at $(1,-1)^{*}$.

For real vectors, $\rho(u, C)$ is determined by the symmetric part of $C$. Because $u^{*} C^{*} u=u^{*} C u$, it follows that

$$
u^{*} C u=u^{*}\left(\frac{C+C^{*}}{2}\right) u+i u^{*}\left(\frac{C-C^{*}}{2 i}\right) u=\frac{1}{2} u^{*}\left(C+C^{*}\right) u
$$

Thus $\rho$ is stationary at real $u$ if and only if $u$ is an eigenvector of $C+C^{*}$.

Minimal Residual. Given $u \neq 0$, then, for any scalar $\rho$

$$
\|(C-\mu) u\|^{2} \geqq\|C u\|^{2}-\|\rho(u) u\|^{2},
$$

with equality only when $\mu=\rho=\rho(u)$.

This classic result can be generalized to the case when $u$ is a rectangular matrix and also to the infinity norm. See Kahan [5a] and Stewart [11] for more details.

Proof.

$$
\begin{aligned}
\|(C-\mu) u\|_{2}^{2} & =u^{*} u|\mu|^{2}-\bar{\mu} u^{*} C u-\mu u^{*} C^{*} u+u^{*} C^{*} C u \\
& =u^{*} u\left\{(\bar{\mu}-\bar{\rho})(\mu-\rho)-|\rho|^{2}+u^{*} C^{*} C u / u^{*} u\right\} \\
& \geqq\|C u\|_{2}^{2}-|\rho|^{2}\|u\|_{2}^{2},
\end{aligned}
$$

with equality only when $\mu=\rho$. 
Corollary 1. $\left\|u^{*}(C-\mu)\right\|^{2} \geqq\left\|u^{*} C\right\|^{2}-|\rho|^{2}\left\|u^{*}\right\|^{2}$ and equality holds only when $\mu=\rho$.

CoRollary 2. $u$ is orthogonal to $(C-\rho(u)) u$.

Note that this property holds for any $C$.

Corollary 3. Let $P_{j}$ denote the set of all monic polynomials of degree $j$ $\left(t^{j}+\cdots\right)$. Then $\psi_{1}(t)=t-\rho(u)$ is the minimizing polynomial of degree 1 for $u$;

$$
\left\|\psi_{1}(C) u\right\| \leqq\|\phi(C) u\|, \quad \phi \in P_{1} .
$$

The minimizing polynomial of degree two is important in practice and is discussed in [1], [5a], and [5c].

4. The Iteration. Lord Rayleigh observed that $\lambda_{j}=\rho\left(x_{j}\right)$ and suggested a scheme for improving on a given approximation $v_{0}$ to $x_{j}$ when $C^{*}=C$. The technique is a variant of inverse iteration and is well defined for all $C$.

Pick a unit vector $v_{0}$. Then, for $k=0,1,2, \cdots$,

(i) form $\rho_{k}=\rho\left(v_{k}\right)=v_{k}^{*} C v_{k}$,

(ii) if $C-\rho_{k}$ is singular, then solve $\left(C-\rho_{k}\right) v_{k+1}=0$ for

(RQI) $v_{k+1} \neq 0$ and halt, otherwise,

(iii) solve $\left(C-\rho_{k}\right) w_{k+1}=v_{k}$,

(iv) normalize $v_{k+1}=w_{k+1} /\left\|w_{k+1}\right\|$.

For purposes of analysis, it is convenient to combine (iii) and (iv) into (iii)' solve $\left(C-\rho_{k}\right) v_{k+1}=v_{k} \tau_{k}$,

where $\tau_{k}$ is the positive scalar which ensures that $\left\|v_{k+1}\right\|=1$. Thus, in terms of $v_{k}$,

$$
\text { (v) } \tau_{k}=\left\|\left(C-\rho_{k}\right)^{-1} v_{k}\right\|^{-1} .
$$

The sequence $\left\{\rho_{k}, v_{k}\right\}$ is called the Rayleigh sequence generated by $v_{0}$ on $C$.

Of course, if $v_{k} \rightarrow x_{1}$ as $k \rightarrow \infty$, then $\rho\left(v_{k}\right) \rightarrow \lambda_{1}$ by the continuity of $\rho$. Consequently, the RQI can be regarded as a method to find eigenvalues or eigenvectors or both.

5. Invariance Properties of RQI. Let $\left\{\rho_{k}, v_{k}\right\}$ be the Rayleigh sequence generated from $v_{0}$ by the RQI on the matrix $C$.

Scaling. The matrix $\alpha C, \alpha \neq 0$, produces the same sequence as $C$.

Translation. The matrix $C-\alpha$ produces the sequence $\left\{\rho_{k}-\alpha, v_{k}\right\}$.

Unitary Similarity. The matrix $Q C Q^{*}, Q$ unitary, produces the sequence $\left\{\rho_{k}, Q v_{k}\right\}$.

Consequently, the iteration has the same form in a new orthonormal coordinate system. In other words, the process is coordinate free, in contrast to the QR algorithm.

6. Local Convergence. It was observed that, when $C=C^{*}$, convergence of the $v_{k}$ to an eigenvector was ultimately very rapid. In 1958 and 1959, 
Ostrowski [4] produced a sequence of six papers in which various aspects of the iteration were analyzed in great detail; he is credited with the first rigorous proof that the asymptotic convergence rate, as $k \rightarrow \infty$, is cubic.

THEOREM. If $C$ is normal and $v_{k} \rightarrow x_{1}$, an eigenvector for $\lambda_{1}$, as $k \rightarrow \infty$, then

$$
\begin{array}{ll}
\text { either }\left\|v_{k+1}-x_{1}\right\| /\left\|v_{k}-x_{1}\right\|^{3} \rightarrow 1 & \text { (the usual case) } \\
\text { or } 0<\left\|v_{k+1}-x_{1}\right\| /\left\|v_{k}-x_{1}\right\|^{3}<1 \quad & \text { for all sufficiently large } k \\
& \text { (the exceptional case) } .
\end{array}
$$

Proof. Let $\mathscr{M}_{1}$ be the eigenspace for $\lambda_{1}$. We may assume that the iteration does not terminate at a finite $k$. Thus, $\rho_{k}$ is never an eigenvalue and both $C$ and $\left(C-\rho_{k}\right)^{-1}$ act like scalars on $\mathscr{M}_{1}$. Consequently, if the projection of $v_{0}$ onto $\mathscr{M}_{1}$ is proportional to $x_{1}$, then so is the projection of $v_{k}$ for all $k$. Therefore, given any small $\epsilon>0$, there is a $k$ such that $v_{k}$ is proportional to $x_{1}+\epsilon u$, where $u$ is a unit vector orthogonal to $\mathscr{M}_{1} ; u=u(k)$.

By the stationary property for normal $C$,

$$
\rho_{k}-\lambda_{1}=\epsilon^{2} u^{*}\left(C-\lambda_{1}\right) u+O\left(\epsilon^{4}\right) \text {. }
$$

By (iii) ${ }^{\prime}, v_{k+1}$ is proportional to $\left(C-\rho_{k}\right)^{-1} v_{k}$ and

$$
\begin{aligned}
\left(C-\rho_{k}\right)^{-1}\left(x_{1}+\epsilon u\right) & =\left(\lambda_{1}-\rho_{k}\right)^{-1}\left[x_{1}+\epsilon\left(\lambda_{1}-\rho_{k}\right)\left(C-\rho_{k}\right)^{-1} u\right] \\
& =\left(\lambda_{1}-\rho_{k}\right)^{-1}\left[x_{1}-\epsilon^{3} u^{*}\left(C-\lambda_{1}\right) u\left(C-\rho_{k}\right)^{-1} u+O\left(\epsilon^{5}\right)\right] .
\end{aligned}
$$

Of course, $\left(C-\rho_{k}\right)^{-1}$ is unbounded, but since $u \in \mathscr{M}_{1}{ }^{\perp},\left\|\left(C-\rho_{k}\right)^{-1} u\right\| \leqq$ $2 / \delta$ for large enough $k$. Here, $\delta=\min \left|\lambda_{1}-\lambda_{j}\right|$ over all $\lambda_{j}$ distinct from $\lambda_{1}$. This establishes the cubic nature of the convergence.

More can be said however. Let $u_{k}$ be the normalized projection of $v_{k}$ onto $\mathscr{M}_{1}^{\perp}$. Thus, $v_{k}=\left(1-\epsilon_{k}^{2}\right)^{1 / 2} x_{1}+\epsilon_{k} u_{k}$. Then $u_{k} \equiv u=\alpha_{k} \prod_{i=0}^{k-1}\left(C-\rho_{i}\right)^{-1} u_{0}$ where $\alpha_{k}$ is a normalizing constant. Usually, $u_{k}$ will converge to $x_{2}$, where $\lambda_{2}$ is the unique eigenvalue closest to $\lambda_{1}$. In this case,

$$
u^{*}\left(C-\lambda_{1}\right) u\left(C-\rho_{k}\right)^{-1} u \rightarrow\left(\lambda_{2}-\lambda_{1}\right)\left(\lambda_{2}-\lambda_{1}\right)^{-1} x_{2}=x_{2}
$$

and convergence is cubic.

However, if there are several distinct eigenvalues closest to $\lambda_{1}$, let $\mathscr{M}_{2}$ be the invariant subspace generated by their eigenvectors. If, by chance, $u_{k}$ converges, then we have the case treated above. Otherwise, as $k \rightarrow \infty$,

$$
u_{k}=\alpha_{k} \prod_{i=0}^{k-1}\left(C-\rho_{i}\right)^{-1} u_{0} \sim \beta_{k}\left(C-\lambda_{1}\right)^{-k} u_{0}
$$

will approach $\mathscr{M}_{2}$ by the theory of the power method. For each $w \equiv \sum_{j=2}^{r} x_{j} \gamma_{j}$ in $\mathscr{M}_{2}$,

$$
\begin{gathered}
\left(C-\lambda_{1}\right) w=\delta \sum_{j=2}^{r} \exp \left(i \theta_{j}\right) x_{j} \gamma_{j} \\
\left(C-\lambda_{1}\right)^{-1} w=\delta^{-1} \sum_{j=2}^{r} \exp \left(-i \theta_{j}\right) x_{j} \gamma_{j}
\end{gathered}
$$


Since $w$ cannot be parallel to $\left(C-\lambda_{1}\right) w$ in this case $\left(\gamma_{2} \gamma_{3} \neq 0\right)$

$$
\begin{aligned}
\left|w^{*}\left(C-\rho_{k}\right) w\right|\left\|\left(C-\rho_{k}\right)^{-1} w\right\| & >\left\|\left(C-\rho_{k}\right) w\right\| \mid\left(C-\rho_{k}\right)^{-1} w \| \\
& \rightarrow\left\|\left(C-\lambda_{1}\right) w\right\|\left\|\left(C-\lambda_{1}\right)^{-1} w\right\| \\
& =1 \text { for } w \text { in } \mathscr{M}_{2} .
\end{aligned}
$$

Moreover, since $w$ lies in $\mathscr{M}_{1}^{\perp}, w^{*}\left(C-\rho_{k}\right) w=\rho(w)-\rho_{k} \neq 0$. By continuity, for large enough $k$,

$$
0<\left|u^{*}\left(C-\lambda_{1}\right) u\right| \cdot\left\|\left(C-\rho_{k}\right)^{-1} u\right\|<1 .
$$

For still larger values of $k$,

$$
0<\left\|v_{k+1}-x_{1}\right\| /\left\|v_{k}-x_{1}\right\|^{3}<1 \text {, too. }
$$

Thus, convergence is even faster in the exceptional case, but the asymptotic behavior is less simple than in the normal case.

7. Fixed Points (for Normal Matrices). It is easily verified that step (ii) of the algorithm ensures that each eigenvector $x_{j}$ of any matrix $C$ is a fixed point of the iteration. These are 'attractive' fixed points. For normal $C$, this fact follows from the local convergence theorem.

For Hermitian matrices, Ostrowski describes a finite neighborhood (unknown a priori) around each eigenvector. When the RQI is started in this region, convergence will occur to the associated eigenvector. These are the only fixed points of the vector iteration. The proof is left to the reader.

Even in the Hermitian case, the $\lambda_{i}$ are not the only fixed points of the scalar sequences $\left\{\rho_{k}\right\}$. If $C x_{j}=\lambda_{j} x_{j}, j=1,2$, then, since $x_{1}^{*} C x_{2}=0$,

$$
\rho\left(x_{1}+x_{2}\right)=\rho\left(x_{1}-x_{2}\right)=\left(\lambda_{1}+\lambda_{2}\right) / 2 .
$$

If $v_{0}=x_{+}=\left(x_{1}+x_{2}\right) / \sqrt{2}$, then it is easily verified that

$$
\rho_{k}=\left(\lambda_{1}+\lambda_{2}\right) / 2, \quad v_{2 k}=x_{+}, \quad v_{2 k+1}=\left(x_{1}-x_{2}\right) / \sqrt{2} \equiv x_{-} .
$$

These mean values are called repulsive fixed points by Ostrowski because any perturbation of $v_{0}$ from $u_{+}$in the $\left(x_{1}, x_{2}\right)$ plane causes $\left\{\rho_{k}\right\}$ to diverge away from the mean value. Note that $x_{+}, x_{-}$are, respectively, the interior and exterior bisectors of the angle between $x_{1}$ and $x_{2}$.

We state without proof that these facts about the fixed points, which were proved for the Hermitian case, continue to hold when $C$ is normal.

It is difficult to describe, in terms of $C$ 's eigenvalues and eigenvectors, those nontrivial $v_{0}$ whose Rayleigh sequences $\left\{v_{k}\right\}$ fail to converge.

8. Global Convergence (Normal Matrices). Although convergence of the RQI had been established rigorously (by Ostrowski) only for starting vectors in definite small neighborhoods of eigenvectors, users found that it was impossible to make the method fail in practice.

In 1966, Kahan had a proof that for Hermitian matrices the RQI converges for almost all starting vectors. This result was not published until 1968, in 
[5b]. Wilkinson [9] also has a proof of this result but couched in terms of the QR tridiagonal algorithm.

Kahan's proof makes use of the observation that the residuals $r_{k}=\left(C-\rho_{k}\right) v_{k}$ are monotonic decreasing in norm. If the limit of these norms is 0 , then $v_{k}$ converges (cubically) tc an eigenvector. If the limit is positive, then the $v_{k}$ do not converge although $\rho_{k}$ converges (linearly) to the mean of a pair of eigenvalues.

Kahan's proof does not make explicit use of the minimal residual property. In fact, the proof exploited symmetry so artfully that it was difficult to see how the result could be extended to non-Hermitian matrices. However, the results of $H$. J. Buurema on the QR algorithm [1] suggested to us that it is the minimal residual property of the Rayleigh Quotient which secures the good global convergence properties of RQI.

THEOREM. Let $r_{k}=\left(C-\rho_{k}\right) v_{k}$ be the residual at the kth step of RQI. If $C$ is normal, then the sequence $\left\{\left\|r_{k}\right\|, k=0,1, \cdots\right\}$ is monotone decreasing for all starting vectors $v_{0}$.

Proof.

$$
\begin{aligned}
\left\|r_{k+1}\right\| & =\left\|\left(C-\rho_{k+1}\right) v_{k+1}\right\| \\
& \leqq\left\|\left(C-\rho_{k}\right) v_{k+1}\right\|, \quad \text { by the minimal residual property, } \\
& =\left|v_{k}^{*}\left(C-\rho_{k}\right) v_{k+1}\right|, \quad \text { since }\left(C-\rho_{k}\right) v_{k+1} \text { is parallel to } v_{k} \\
& \leqq\left\|v_{k}^{*}\left(C-\rho_{k}\right)\right\|\left\|v_{k+1}\right\|, \quad \text { by the Cauchy-Schwarz inequality, } \\
& =\left\|v_{k}^{*}\left(C-\rho_{k}\right)\right\|, \quad \text { since } v_{k+1} \text { is a unit vector, } \\
& =\left\|\left(C-\rho_{k}\right) v_{k}\right\|, \quad \text { since } C \text { is normal, } \\
& =\left\|r_{k}\right\| .
\end{aligned}
$$

Equality can occur only if $\rho_{k+1}=\rho_{k}$ and $v_{k+1}^{*}$ is parallel to $v_{k}^{*}\left(C-\rho_{k}\right)$.

Various generalizations suggest themselves and we will turn to them in Section 10.

The rest of the proof that Kahan's result holds for normal matrices follows the original line of thinking but is more complicated.

THEOREM. Let the RQI be applied to a normal matrix $C$ with starting vector $v_{0}$. As $k \rightarrow \infty$,

(i) $\rho_{k}=\rho\left(v_{k}\right)$ converges, and either

(ii) $\left(\rho_{k}, v_{k}\right)$ converges to an eigenpair $(\lambda, x)$ (and the asymptotic rate is cubic), or

(iii) $\rho_{k}$ converges (linearly) to a point equidistant from $s(\geqq 2)$ eigenvalues of $C$, and the sequence $\left\{v_{k}\right\}$ cannot converge. It may or may not have a limit cycle.

Proof. By the above theorem, the monotone sequence $\left\{\left\|r_{k}\right\|\right\}$ is bounded below by 0 . Let its limit be $\tau \geqq 0$ and let $\theta_{k}$ be the acute angle between $v_{k+1}$ and $\left(C-\rho_{k}\right)^{*} v_{k}$. Note that

$$
\begin{aligned}
\left\|r_{k+1}\right\|^{2} & =\left\|\left(C-\rho_{k}\right) v_{k+1}\right\|^{2}+2 \operatorname{Real}\left[\left(\overline{\rho_{k}-\rho_{k+1}}\right) v_{k+1}^{*}\left(C-\rho_{k}\right) v_{k+1}\right]+\left|\rho_{k}-\rho_{k+1}\right|^{2} \\
& =\left|v_{k}^{*}\left(C-\rho_{k}\right) v_{k+1}\right|^{2}-\left|\rho_{k}-\rho_{k+1}\right|^{2} \\
& =\left\|r_{k}\right\|^{2} \cos ^{2} \theta_{k}-\left|\rho_{k}-\rho_{k+1}\right|^{2} .
\end{aligned}
$$


Since $\left\|r_{k}\right\| \rightarrow \tau$, we must have $\rho_{k}-\rho_{k+1} \rightarrow 0$ as $k \rightarrow \infty$. Moreover, the $\rho_{k}$ are confined to the numerical range of $C$, a closed, bounded region in the complex plane. Therefore, the sequence $\left\{\rho_{k}\right\}$ has limit points ${ }^{* *}$ and, since $\rho_{k}-\rho_{k+1} \rightarrow 0$, there can only be one of them. Thus,

$$
\rho_{k} \rightarrow \rho \text { as } k \rightarrow \infty \text {. }
$$

Since the $v_{k}$ are confined to the unit sphere in complex $n$-space, the sequence $\left\{v_{k}\right\}$ has limit points too. The problem is, how many and what are they?

Case 1. $\tau=0$. Take a subsequence of $\left\{v_{k}\right\}$ converging to a limit point $v$. Taking limits in this subsequence yields

$$
\|(C-\rho) v\|=\lim \left\|\left(C-\rho_{k}\right) v_{k}\right\|=0 .
$$

Thus, $\rho$ is an eigenvalue and each limit point $v$ is one of $\rho$ 's eigenvectors. The local convergence theorem shows that the only limit point is the spectral projection of $v_{0}$ onto $\rho$ 's eigenspace. Thus, $\left(\rho_{k}, v_{k}\right)$ converges to some eigenpair $(\lambda, x)$ of $C$. We have already seen that, when $C$ is normal, the asymptotic rate is cubic.

Case 2. $\tau>0$. As $k \rightarrow \infty$,

$$
\cos ^{2} \theta_{k}=\left(\left\|r_{k+1}\right\|^{2}+\left|\rho_{k}-\rho_{k+1}\right|^{2}\right) /\left\|r_{k}\right\|^{2} \rightarrow 1 .
$$

So $\tau_{k}=\left|v_{k}^{*}\left(C-\rho_{k}\right) v_{k+1}\right|=\left\|r_{k}\right\| \cos \theta_{k} \rightarrow \tau$. Now

$$
\begin{aligned}
\tau_{k}^{2} & =v_{k+1}^{*}\left(C-\rho_{k}\right) *\left(C-\rho_{k}\right) v_{k+1} \\
& \leqq\left\|v_{k+1}^{*}\right\|\left\|\left(C-\rho_{k}\right) *\left(C-\rho_{k}\right) v_{k+1}\right\|, \quad \text { by Cauchy-Schwarz, } \\
& =\left\|\left(C-\rho_{k}\right){ }^{*} v_{k} \tau_{k}\right\|, \text { since }\left(C-\rho_{k}\right) v_{k+1}=v_{k} \tau_{k}, \\
& =\tau_{k}\left\|r_{k}\right\|, \text { by normality. }
\end{aligned}
$$

Consequently, equality in the Cauchy-Schwarz inequality must hold in the limit, as $k \rightarrow \infty$. Let $K$ be a subsequence of $\{0,1,2, \cdots\}$ such that $\lim _{k \in K} v_{k}=v$, a limit point. Taking this limit shows that $(C-\rho)^{*}(C-\rho) v$ is proportional to $v$. Since

$$
\|(C-\rho) v\|=\tau,
$$

we have

$$
(C-\rho)^{*}(C-\rho) v=\tau^{2} v
$$

for each limit point. Using the same subsequence again, we find

$$
\rho=\lim _{k \in K} \rho\left(v_{k}\right)=\rho(v) .
$$

These last three relations $(\mathrm{X}),(\mathrm{Y}),(\mathrm{Z})$ characterize the limit points $v$ of $\left\{v_{k}\right\}$. Each is a singular vector of $C-\rho$ with associated singular value $\tau$. Note that $\tau=\|(C-\rho) v\|$.

When $C$ is normal, we can describe the $v$ in terms of $C$ 's spectral decomposition because the singular values of $C-\rho$ are simply $\left\{\left|\lambda_{i}-\rho\right|, i=1, \cdots, n\right\}$.

\footnotetext{
** Since these limit points are means of subsets of $C$ 's eigenvalues (see Case 2) they are finite in number.
} 
Let $\Gamma$ be the subset of eigenvalues $\lambda_{i}$ satisfying $\left|\lambda_{i}-\rho\right|=\tau$ and let $m$ be the number of distinct eigenvalues in $\Gamma$.

We now show that $m$ must be greater than one. Suppose to the contrary that $m=1$ and $\lambda_{j}=\rho+\tau e^{i \theta}$, then $\tau^{2}$ is a simple eigenvalue of $(C-\rho)^{*}(C-\rho)$ and, since $(C-\rho)^{*}(C-\rho) x_{j}=\tau^{2} x_{j}$, we would have $v=e^{i \phi} x_{j}$ and $v^{*} C v=$ $\rho+e^{i \theta} \tau$ which contradicts $\rho(v)=\rho$. Thus $2 \leqq m \leqq n$.

Consequently, $\tau$ is a multiple singular value of $C-\rho$ and

$$
\begin{aligned}
& v=\sum \alpha_{j} x_{j}, \quad j \in \Gamma, \\
& 1=\sum\left|\alpha_{j}\right|^{2} .
\end{aligned}
$$

Moreover, since $\lambda_{j}-\rho=\tau e^{i \theta,}, j \in \Gamma$,

$$
0=v^{*}(C-\rho) v=\tau \sum\left|\alpha_{j}\right|^{2} e^{i \theta,} .
$$

Thus, $\rho$ is a weighted mean of the equidistant eigenvalues and the vectors

$$
\sum \alpha_{j} e^{-i k \theta_{1}} x_{j}, \quad j \in \Gamma,
$$

are limit vectors for each $k=0,1,2, \cdots$. This set will be finite if the $\theta_{j}$ are rational multiples of $\pi$.

Definition. Let $\Delta$ be the subspace spanned by $\left\{x_{j}, j \in \Gamma\right\}$.

Corollary. If $C^{*}=C$, then, in case (iii), $\rho=\left(\lambda_{p}+\lambda_{q}\right) / 2$ and the only limit vectors are $\left(x_{p} \pm x_{q}\right) / \sqrt{2}$.

Proof. If $C^{*}=C$, then the $\lambda_{j}$ must be real. So $m=2, \theta_{1}=0, \theta_{2}=\pi$. Consequently, $\left|\alpha_{1}\right|^{2}=\left|\alpha_{2}\right|^{2}=\frac{1}{2}$. Thus, $v_{k}$ tends to oscillate between the internal and external bisectors of $x_{p}$ and $x_{q}$. Of course, $\lambda_{p}$ and $\lambda_{q}$ could be multiple but $x_{p}, x_{q}$ are the unique eigendirections in the plane defined by the projection of $v_{0}$ and $v_{1}$ onto $\Delta$.

9. Instability of Case (iii). Let $z$ be any unit vector in the invariant subspace $\Delta$ defined above. Thus, with the notation of Section 8 ,

$$
z=\sum \xi_{j} x_{j}, \quad j \in \Gamma,
$$

with

$$
1=\sum\left|\xi_{j}\right|^{2} \text { and } \rho(z)=\left[\rho \sum\left|\xi_{j}\right|^{2}+\tau \sum e^{i \theta}\left|\xi_{j}\right|^{2}\right] / \sum\left|\xi_{j}\right|^{2} .
$$

Differentiating, with respect to $\left|\xi_{j}\right|^{2}$, we find

$$
\partial \rho(z) / \partial\left|\xi_{j}\right|^{2}=\rho+\tau e^{i \theta_{1}}-\rho(z) .
$$

The special values $\alpha_{j}$ of $\xi_{j}$ belonging to the limit points satisfy $0=\sum\left|\alpha_{j}\right|^{2} e^{i \theta,}$ and $\rho(v)=\rho$. So

$$
\partial \rho / \partial\left|\alpha_{j}\right|^{2}=e^{i \theta_{,}} \tau \neq 0,
$$

for each $j$.

So an increase in an $\left|\alpha_{j}\right|^{2}$ pushes the Rayleigh Quotient from $\rho$ towards $\lambda_{j}=\rho+\tau e^{i \theta}$. Almost all perturbations in $\Delta$ of a limit point $v$ generate Rayleigh sequences which converge to an eigenvector. 
10. The RQI for Nonnormal Matrices. The stationary property of the Rayleigh Quotient fails for nonnormal matrices and, consequently, the asymptotic convergence rate can be, at best, quadratic. Secondly, although the minimal residual property persists, the norms of the residuals of a Rayleigh sequence are not always monotonic decreasing. This bars us from extending the approach of the previous sections to obtain global convergence properties.

Nevertheless, except for the unstable configurations noted in the theorems, the RQI still seems to converge for nonnormal matrices, albeit less rapidly than in the normal case.

An ideal would be a generalization of RQI which, for all matrices,

(i) was simple to execute,

(ii) enjoyed the stationarity property at eigenvectors,

(iii) generated monotonically decreasing residuals.

This has not been done. Ostrowski proposes a two-sided process satisfying (i) and (ii) and having local superquadratic convergence for nondefective matrices. However, unfortunate choices of initial vectors can provoke a breakdown of the process. In contrast, we investigate here a generalization of RQI possessing properties (i) and (iii). It converges, though slowly, for almost all starting vectors, even for nondefective matrices.

Theoretically, then, a judicious application of both methods can produce convergence at a satisfactory rate in almost all cases.

Even better would be a new idea which yielded a convergence proof for the standard RQI for almost all starting vectors.

11. Ostrowski's Two-Sided Iteration. Examination of the proof of stationarity in the normal case shows that it depends on the fact that, for eigenvectors $v, v^{*} C=\lambda v^{*}$ as well as $C v=\lambda v$. To preserve this property in general, Ostrowski makes the row vector different from the column vector.

Definition. $\rho\left(v^{*}, u\right) \equiv \rho\left(v^{*}, u, C\right) \equiv v^{*} C u / v^{*} u$ provided that $v^{*} u \neq 0$.

Homogeneity and translation invariance persist, but the boundedness property fails.

Stationarity. $\rho$ is stationary at $\left(v^{*}, u\right)$ if and only if $v^{*}$ and $u$ are, respectively, row and column eigenvectors of $C$ with eigenvalue $\rho$ and $v^{*} u \neq 0$.

Proof. Let $\rho=\rho\left(v^{*}, u\right)$ and $\Delta=(v+\epsilon w)^{*}(u+\eta z) \neq 0$.

$$
\rho\left(v^{*}+\epsilon w^{*}, u+\eta z\right)-\rho=\left\{\epsilon w^{*}(C-\rho) u+\eta v^{*}(C-\rho) z+\epsilon \eta w^{*}(C-\rho) z\right\} / \Delta .
$$

This is $O(\epsilon \eta)$ for all $w^{*}$ and $z$ if and only if $(C-\rho) u=0, v^{*}(C-\rho)=0^{*}$.

The Iteration. Pick initial vectors $v_{0}^{*}$ and $u_{0}$ satisfying $v_{0}^{*} u_{0} \neq 0,\left\|v_{0}\right\|=$ $\left\|u_{0}\right\|=1$. For $k=0,1,2, \cdots$,

(i) compute $\rho_{k}=\rho\left(v_{k}^{*}, u_{k}\right)$,

(ii) if $C-\rho_{k}$ is singular solve $y^{*}\left(C-\rho_{k}\right)=0$ and $\left(C-\rho_{k}\right) x=0$ for $y^{*}$, $x \neq 0$ and stop, otherwise

(iii) solve both $v_{k+1}^{*}\left(C-\rho_{k}\right)=v_{k}^{*} \nu_{k}, \quad\left(C-\rho_{k}\right) u_{k+1}=u_{k} \tau_{k}$, where $\nu_{k}$ and $\tau_{k}$ are normalizing factors. 
(iv) if $v_{k+1}^{*} u_{k+1}=0$, then stop and admit failure.

Local Convergence. If $v_{k}^{*} \rightarrow y^{*}, \quad u_{k} \rightarrow x$ as $k \rightarrow \infty$ where $y^{*}(C-\lambda)=0^{*}$, $(C-\lambda) x=0, y^{*} x \neq 0,\|x\|=\left\|y^{*}\right\|=1$, then $\rho_{k} \rightarrow \lambda$ and the asymptotic convergence rate is cubic.

Proof. Since $y^{*} x \neq 0$, the eigenvalue $\lambda$ must correspond to linear elementary divisors. Even if $\lambda$ is multiple, the spectral projection of $u_{0}$, and all $u_{k}$, on $\lambda$ 's column eigenspace $N_{\lambda}$ is in the direction of $x$. Likewise, the spectral projection of $v_{0}^{*}$, and all $v_{k}^{*}$, onto $\lambda$ 's row space $M_{\lambda}$ is in the direction of $y^{*}$. Moreover, $N_{\lambda}$ and $M_{\lambda}$ each have unique invariant complements $N_{\lambda}^{c}$ and $M_{\lambda}^{c}$, respectively. The point of this is that we can write $u_{k}=\alpha(x+\epsilon z), v_{k}^{*}=$ $\beta\left(y^{*}+\eta w^{*}\right)$ uniquely where $\alpha, \epsilon, z, \beta, \eta, w^{*}$ all depend on $k ; \alpha, \beta$ are normalizing factors, and $z$ and $w^{*}$ are unit vectors in $N_{\lambda}^{c}$ and $M_{\lambda}^{c}$ respectively. By hypothesis, $\epsilon \rightarrow 0, \eta \rightarrow 0$, as $k \rightarrow \infty$.

Now we can imitate the proof for the normal case:

$$
\begin{aligned}
\rho & =\rho_{k}=\rho\left(y^{*}+\eta w^{*}, x+\epsilon z\right)=\lambda+\epsilon \eta w^{*}(C-\lambda) z / \Delta, \\
\Delta & =\Delta_{k}=y^{*} x+\epsilon \eta w^{*} z, \quad y^{*} x \neq 0, \\
v_{k+1}^{*} & =\nu\left(y^{*}+\eta w^{*}\right)(C-\rho)^{-1}=\left[y^{*}+\eta(\lambda-\rho) w^{*}(C-\rho)^{-1}\right] \beta_{k+1}, \\
u_{k+1} & =(C-\rho)^{-1}(x+\epsilon z) \tau=\left[x+\epsilon(\lambda-\rho)(C-\rho)^{-1} z\right] \alpha_{k+1} .
\end{aligned}
$$

As $\rho \rightarrow \lambda$, the operator $(C-\rho)^{-1}$ becomes unbounded. However, we are only interested in applying $(C-\rho)^{-1}$ to vectors in $N_{\lambda}^{c}$ and $M_{\lambda}^{c}$. Since these are invariant subspaces, we may let $R(\rho)$ be the resolvent of $C$ restricted to $N_{\lambda}^{c}$ (on the right) and $M_{\lambda}^{c}$ (on the left). Because the spectrum of $C$ is finite, $R(\lambda)$ is a bounded operator independent of $k$. When $C$ is normal, $\|R(\lambda)\|=$ $\left(\min _{\lambda_{j} \neq \lambda}\left|\lambda_{j}-\lambda\right|\right)^{-1}$ but, in general, it may be much larger than that.

By the continuity of the operator (or lub) norm $\|C\| \equiv \max \|C u\| /\|u\|$, we have, for all large enough $k$,

$$
\begin{gathered}
\left\|w_{k}^{*}\left(C-\rho_{k}\right)^{-1}\right\|<2\left\|w_{k}^{*}(C-\lambda)^{-1}\right\| \leqq 2\|R(\lambda)\|, \\
\left\|\left(C-\rho_{k}\right)^{-1} z\right\|<2\left\|(C-\lambda)^{-1} z\right\| \leqq 2\|R(\lambda)\| .
\end{gathered}
$$

Thus, from the expressions for $u_{k}=\alpha_{k}\left(x+\epsilon_{k} v_{k}\right)$, we have

$$
\begin{aligned}
\epsilon_{k+1} v_{k+1} & =\epsilon_{k}\left(\lambda-\rho_{k}\right)\left(C-\rho_{k}\right)^{-1} z_{k}, \\
\epsilon_{k+1} & \leqq \epsilon_{k}\left|\lambda-\rho_{k}\right|\|R(\lambda)\| \\
& \leqq \epsilon_{k}^{2} \eta_{k} \Gamma\|R(\lambda)\|, \quad \text { by stationarity. }
\end{aligned}
$$

Similarily, $\eta_{k+1} \leqq \eta_{k}^{2} \epsilon_{k} \Gamma\|R(\lambda)\|$ and this is cubic convergence.

Remark 1. In his third paper on RQI, Ostrowski proved that convergence was superquadratic and established its cubic nature under special hypotheses, such as $\lambda$ real and $C$ nondefective. His approach was more analytic and less geometric than ours.

Remark 2. If, in the above theorem, $y^{*} x=0$ (defective case), convergence is still possible but the asymptotic rate is linear.

Remark 3. In his fifth paper on the RQI, Ostrowski does an injustice to 
his own algorithm. In assessing efficiency, he uses the solution of a system of linear equations as the unit of work. With this measure, his two-sided algorithm is twice as costly as the standard RQI. However, if a triangular factorization is used as the unit, then Ostrowski's algorithm costs no more than RQI.

Remark 4. The danger in this algorithm is that $v_{0}^{*}$ and $u_{0}$ could be very nearly eigenvectors but belong to different eigenvalues. Hence the poor global qualities for independent $u_{0}$ and $v_{0}$.

12. The Alternating Rayleigh Quotient Iteration. Here is a different generalization designed to converge globally.

The Iteration. Pick a unit starting vector $z_{0}$ and then, for $k=0,2,4, \cdots$,

(a) form $\rho_{k}=\rho\left(z_{k}\right)$,

(b) solve $z_{k+1}^{*}\left(C-\rho_{k}\right)=\tau_{k} z_{k}^{*}$ such that $\left\|z_{k+1}^{*}\right\|=1$,

(c) form $\rho_{k+1}=\rho\left(z_{k+1}\right)$,

(d) solve $\left(C-\rho_{k+1}\right) z_{k+2}=z_{k+1} \tau_{k+1}$ such that $\left\|z_{k+2}\right\|=1$.

If by chance $C-\rho_{k}$ or $C-\rho_{k+1}$ is singular, solve the homogeneous system for the associated eigenvector. This scheme is designed to obviate the need for normality in the original proof of monotonicity of residuals. When $C^{*}=C$, the original RQI is recovered.

Our aim is to have $z_{2 k}$ converge to a column eigenvector and $z_{2 k+1}^{*}$ to its associated row eigenvector as $k \rightarrow \infty$.

Monotonic Residuals.

$$
\begin{aligned}
& \left\|\left(C-\rho_{k+2}\right) z_{k+2}\right\| \leqq\left\|\left(C-\rho_{k}\right) z_{k}\right\|, \\
& \left\|z_{k+1}^{*}\left(C-\rho_{k+1}\right)\right\| \leqq\left\|z_{k-1}^{*}\left(C-\rho_{k-1}\right)\right\|,
\end{aligned}
$$

with equality (for $k$ even) only if

(i) $\rho_{k+2}=\rho_{k+1}=\rho_{k}$, and

(ii) $z_{k+2}^{*} / / z_{k+1}^{*}\left(C-\rho_{k+1}\right), z_{k+1} / /\left(C-\rho_{k}\right) z_{k}$.

Here, $u / / v$ means that $u$ is proportional to $v$.

Proof.

$$
\begin{aligned}
\left\|\left(C-\rho_{k+2}\right) z_{k+2}\right\| & \leqq\left\|\left(C-\rho_{k+1}\right) z_{k+2}\right\|, \quad \text { equality only if } \rho_{k+2}=\rho_{k+1}, \\
& =\left|z_{k+1}^{*}\left(C-\rho_{k+1}\right) z_{k+2}\right|, \quad \text { by }(\mathrm{d}), \\
& \leqq\left\|z_{k+1}^{*}\left(C-\rho_{k+1}\right)\right\|, \quad \text { by the Cauchy-Schwarz inequality } \\
& \leqq\left\|z_{k+1}^{*}\left(C-\rho_{k}\right)\right\|, \quad \text { equality only if } \rho_{k+1}=\rho_{k}, \\
& =\left|z_{k+1}^{*}\left(C-\rho_{k}\right) z_{k}\right|, \quad \text { by (b), } \\
& \leqq\left\|\left(C-\rho_{k}\right) z_{k}\right\|, \quad \text { by the Cauchy-Schwarz inequality } \\
& \left.\quad \text { (equality only if }\left(C-\rho_{k}\right) z_{k} / / z_{k+1}\right) .
\end{aligned}
$$

The proof for the other relationship is similar.

Global Convergence. For all $z_{0}$, as $k \rightarrow \infty$,

(i) $\rho_{k} \rightarrow \rho, z_{2 k} \rightarrow u, z_{2 k+1}^{*} \rightarrow v^{*}$, either 
(ii) $(C-\rho) u=0, v^{*}(C-\rho)=0$, or

(iii) $(C-\rho)^{*}(C-\rho) u=\tau^{2} u,(C-\rho)(C-\rho)^{*} v=\tau^{2} v$, where

$$
\|(C-\rho) u\|=\tau=\left\|v^{*}(C-\rho)\right\| .
$$

Proof. The sequence $\left\{\left\|\left(C-\rho_{2 k}\right) z_{2 k}\right\|, k=0,1,2, \cdots\right\}$ is monotone and bounded below by 0 . Let its limit be $\tau_{+}$. From the theorem on monotonic residuals,

$$
\begin{aligned}
\rho_{k+2}-\rho_{k} & \rightarrow 0, & \rho_{k+1}-\rho_{k} & \rightarrow 0, \\
\theta_{k+1} & \rightarrow 0, & \phi_{k} & \rightarrow 0,
\end{aligned}
$$

where $\theta_{k+1}, \phi_{k}$ are respectively the acute angles between $z_{k+2}^{*}$ and $z_{k+1}^{*}\left(C-\rho_{k+1}\right)$, $z_{k+1}$ and $\left(C-\rho_{k}\right) z_{k}$.

Since $\rho_{k}$ is bounded, the sequence $\left\{\rho_{k}\right\}$ has a finite number of limit points and, since $\rho_{k+1}-\rho_{k} \rightarrow 0$, it must converge to some $\rho$ as $k \rightarrow \infty$.

Case 1. $\tau_{+}=0$. By continuity of the norm, $\|(C-\rho) z\|=0$ for all limit points $z$ of $\left\{z_{2 k}\right\}$. So each such $z$ is an eigenvector for $\rho$ and each limit point of $\left\{z_{2 k+1}^{*}\right\}$ must be a row eigenvector for $\rho$. However, the only possible limit point is the spectral projection of $z_{0}$ (or $\left.z_{0}^{*}\right)$ onto $\rho$ 's eigenspace. This establishes (ii).

In fact, $\rho_{k}$ converges at least as quickly as $\tau_{k}$ since

$$
\frac{\rho_{k+1}-\rho_{k}}{\tau_{k}} \rightarrow u^{*} v= \begin{cases}1 / \operatorname{cond}(\rho), & \text { nondefective, } \\ 0, & \text { defective. }\end{cases}
$$

Here, $\operatorname{cond}(\rho)$ is the secant of the acute angle between the eigenvectors $u$ and $v^{*}$.

Case 2. $\tau_{+}>0$. We find

$$
\begin{array}{rlrl}
z_{k}^{*} z_{k+2} & =z_{k+1}^{*}\left(C-\rho_{k}\right) z_{k+2} / \tau_{k} \\
& =\tau_{k}^{-1} z_{k+1}^{*}\left[\left(C-\rho_{k+1}\right)+\left(\rho_{k+1}-\rho_{k}\right)\right] z_{k+2} \\
& =\tau_{k}^{-1}\left[\tau_{k+1}-\left(\rho_{k+1}-\rho_{k}\right)\left(\overline{\rho_{k+2}}-\overline{\rho_{k+1}}\right) / \tau_{k+1}\right] \\
& \rightarrow 1 . & \text { since } z_{k+1}^{*}=z_{k+2}^{*}\left(C-\rho_{k+1}\right) * / \tau_{k+1}
\end{array}
$$

Hence, the unit vectors $z_{k}$ satisfy $\left\|z_{k+2}-z_{k}\right\| \rightarrow 0$ for $k=0,2,4$. In this case, too, $z_{2 k}$ converges as $k \rightarrow \infty$.

Let $\tau_{-}$be the limit of the norms of the odd residuals. By reasoning symmetrical to Case 1 , we see that $\tau_{-}=0$ implies $\tau_{+}=0$. Thus, $\tau_{-}>0$ and, consequently, $z_{2 k+1}^{*}$ converges too. Let

Moreover,

$$
z_{2 k} \rightarrow u, \quad z_{2 k+1}^{*} \rightarrow v^{*} .
$$

$$
\begin{aligned}
& \tau_{+}=\lim z_{k+1}^{*}\left(C-\rho_{k}\right) z_{k}=v^{*}(C-\rho) u=\tau, \text { say, } \\
& \tau_{-}=\lim z_{k+1}^{*}\left(C-\rho_{k+1}\right) z_{k+2}=v^{*}(C-\rho) u=\tau .
\end{aligned}
$$

Furthermore, 


$$
u^{*} v=\lim z_{k}^{*} z_{k+1}=\lim \left(\rho_{k+1}-\rho_{k}\right) / \tau_{k}=0,
$$

and, as $k \rightarrow \infty$,

$$
\begin{aligned}
z_{k+1} & =\left(C-\rho_{k+1}\right) z_{k+2} / \tau_{k+1} \rightarrow(C-\rho) u / \tau=v, \\
z_{k}^{*} & =z_{k+1}^{*}\left(C-\rho_{k}\right) / \tau_{k} \rightarrow v^{*}(C-\rho) / \tau=u^{*} .
\end{aligned}
$$

This yields (iii); $\tau$ is a multiple singular value of $C-\rho$ and $u$ and $v$ are singular vectors.

Global convergence has been bought at a heavy price. The right-hand sides in (b) and (d) force the monotonicity but prolong the process. Asymptotic convergence of $\rho_{k}$ is linear, at best, for nonnormals, with convergence factor $1-1 / \operatorname{cond}(\rho)^{2}$.

These results suggest that one should begin with this alternating scheme and then (fairly soon) switch to Ostrowski's two-sided algorithm.

\section{Summary.}

\section{TABLe 1}

S: Is $\rho$ stationary at an eigenvector?

M: Are the residuals monotonic decreasing?

\begin{tabular}{|c|c|c|c|c|}
\hline RQI & Symmetric & Normal & Nondefective & Defective \\
\hline Standard & $\begin{array}{l}\text { S: Yes } \\
\text { M: Yes } \\
\text { G: Yes } \\
\text { L: Cubic }\end{array}$ & $\begin{array}{l}\text { S: Yes } \\
\text { M: Yes } \\
\text { G: Yes } \\
\text { L: Cubrc }\end{array}$ & $\begin{array}{l}\text { S: No } \\
\text { M: No } \\
\text { G: ? } \\
\text { L: Quadratic }\end{array}$ & $\begin{array}{l}\text { S: No } \\
\text { M: No } \\
\text { G: ? } \\
\text { L: Linear }\end{array}$ \\
\hline Two-Sided & $\begin{array}{l}\text { Iteration reduces } \\
\text { to the one above } \\
\text { if } u_{0}=v_{0}\end{array}$ & $\begin{array}{l}\text { S: Yes } \\
\text { M: No } \\
\text { G: ? } \\
\text { L: Cubic }\end{array}$ & $\begin{array}{l}\text { S: Yes } \\
\text { M: ? } \\
\text { G: ? } \\
\text { L: Cubic }\end{array}$ & $\begin{array}{l}\text { S: No } \\
\text { M: ? } \\
\text { G: ? } \\
\text { L: Linear }\end{array}$ \\
\hline Alternating & $\begin{array}{l}\text { Iteration reduces } \\
\text { to the one above }\end{array}$ & $\begin{array}{l}\text { S: Yes } \\
\text { M: Yes } \\
\text { G: Yes } \\
\text { L: Cubic }\end{array}$ & $\begin{array}{l}\text { S: No } \\
\text { M: Yes } \\
\text { G: Yes } \\
\text { L: Linear }\end{array}$ & $\begin{array}{l}\text { S: No } \\
\text { M: Yes } \\
\text { G: Yes } \\
\text { L: Harmonic }\end{array}$ \\
\hline
\end{tabular}

G: Does the Rayleigh sequence converge for almost all starting vectors?

$\mathrm{L}$ : What is the asymptotic convergence rate to an eigenvector?

Departments of Mathematics, Electrical Engineering and Computer Science University of California at Berkeley Berkeley, California 94720

1. H. J. BuUrema, A Geometric Proof of Convergence for the QR Method, Report TW-62, Mathematisch Institut, Groningen, North East Netherlands, 1968.

2. S. H. Crandall, "Iterative procedures related to relaxation methods for eigenvalue problems," Proc. Roy. Soc. London Ser. A, v. 207, 1951, pp. 416-423. MR 13, 163. 
3. P. LANCAster, "A generalized Rayleigh quotient iteration for lambda-matrices," Arch. Rational Mech. Anal., v. 8, 1961, pp. 309-322. MR 25 \# 2697.

4. A. M. Ostrowski, "On the convergence of the Rayleigh quotient iteration for the computation of characteristic roots and vectors. I-VI," Arch. Rational Mech. Anal., v. 1-4, 1958/59, pp. 233-241, 423-428, 325-340, 341-347, 472-481, 153-165. MR 21 \# 427; \# 4541a, b; \# 6691; 22 \# 8654.

5a. W. KahaN, Inclusion Theorems for Clusters of Eigenvalues of Hermitian Matrices, Technical Report, Dept. of Computer Sci., University of Toronto, 1967.

5b. B. N. Parlett \& W. Kahan, "On the convergence of a practical QR algorithm. (With discussion)," Information Processing 68 (Proc. IFIP Congress, Edinburgh, 1968), vol. I: Mathematics, Software, North-Holland, Amsterdam, 1969, pp. 114-118. MR 40 \# 8242.

5c. B. Parlett, Certain Matrix Eigenvalue Techniques Discussed from a Geometric Point of View, AERE Report 7168, Theor. Physics Div., AERE, Berkshire, England.

6. LoRd Rayleigh, The Theory of Sound, 2nd rev. ed., Macmillan, New York, 1937.

7. G. TEMPLE, “The accuracy of Rayleigh's method of calculating the natural frequencies of vibrating systems," Proc. Roy. Soc. London Ser. A, v. 211, 1952, pp. 204-224. MR 13, 691.

8. J. H. Wilkinson, The Algebraic Eigenvalue Problem, Clarendon Press, Oxford, 1965. MR 32 \# 1894.

9. J. H. WiLkinson, "Global convergence of tridiagonal QR algorithm with origin shifts," Linear Algebra and Appl., v. 1, 1968, pp. 409-420. MR 38 \# 2938.

10. M. Marcus \& H. Minc, A Survey of Matrix Theory and Matrix Inequalities, Allyn and Bacon, Boston, Mass., 1964. MR 29 \# 112.

11. G. W. Stewart, "Error and perturbation bounds for subspaces associated with certain eigenvalue problems," SIAM J. Numer. Anal. (To appear.) 